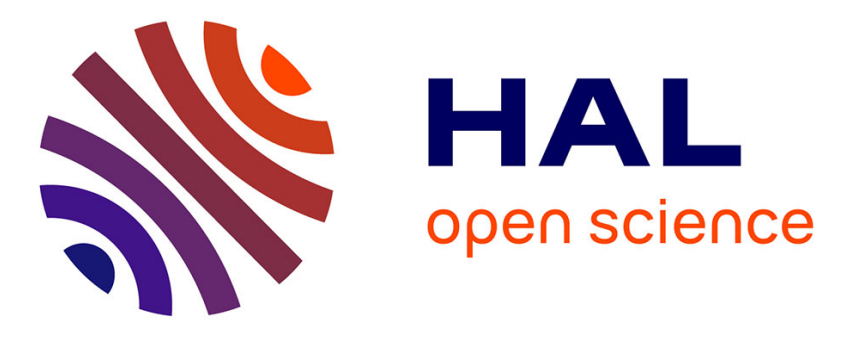

\title{
Transport in ITO Nanocrystals with Short- to Long-Wave Infrared Absorption for Heavy Metal-Free Infrared Photodetection
}

Junling Qu, Clément Livache, Bertille Martinez, Charlie Gréboval, Audrey Chu, Elisa Merrigio, Julien Ramade, Hervé Cruguel, Xiangzhen Xu, Anna Proust, et al.

\section{To cite this version:}

Junling Qu, Clément Livache, Bertille Martinez, Charlie Gréboval, Audrey Chu, et al.. Transport in ITO Nanocrystals with Short- to Long-Wave Infrared Absorption for Heavy Metal-Free Infrared Photodetection. ACS Applied Nano Materials, 2019, 10.1021/acsanm.9b00067 . hal-02043472

\author{
HAL Id: hal-02043472 \\ https://hal.science/hal-02043472
}

Submitted on 31 Oct 2019

HAL is a multi-disciplinary open access archive for the deposit and dissemination of scientific research documents, whether they are published or not. The documents may come from teaching and research institutions in France or abroad, or from public or private research centers.
L'archive ouverte pluridisciplinaire HAL, est destinée au dépôt et à la diffusion de documents scientifiques de niveau recherche, publiés ou non, émanant des établissements d'enseignement et de recherche français ou étrangers, des laboratoires publics ou privés. 


\title{
Transport in ITO Nanocrystals with Short- to Long-Wave Infrared Absorption for Heavy Metal-Free Infrared Photodetection
}

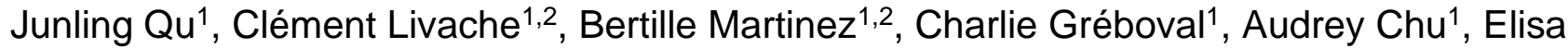
Meriggio ${ }^{1}$, Julien Ramade ${ }^{1}$, Hervé Cruguel ${ }^{1}$, Xiang Zhen $\mathrm{Xu}^{2}$, Anna Proust ${ }^{3}$, Florence Volatron ${ }^{3}$, Grégory Cabailh ${ }^{1}$, Nicolas Goubet ${ }^{1,2,4}$, Emmanuel Lhuillier ${ }^{1^{*}}$

${ }^{1}$ Sorbonne Université, CNRS, Institut des NanoSciences de Paris, INSP, F-75005 Paris, France

'Laboratoire de Physique et d'Etude des Matériaux, ESPCI-Paris, PSL Research University, Sorbonne Université UPMC Univ Paris 06, CNRS, 10 rue Vauquelin 75005 Paris, France.

${ }^{3}$ Sorbonne Université, CNRS, Institut Parisien de Chimie Moléculaire, IPCM, F-75005 Paris, France.

${ }^{4}$ Sorbonne Université, CNRS, Laboratoire de la Molécule aux Nano-objets ; Réactivité, Interactions et Spectroscopies, MONARIS, F-75005 Paris, France

\begin{abstract}
Nanocrystals are often described as interesting materials for the design of low-cost optoelectronic devices especially in the infrared range. However the driving materials reaching infrared absorption are generally heavy metalcontaining $(\mathrm{Pb}$ and $\mathrm{Hg}$ ) with a high toxicity. An alternative strategy to achieve infrared transition is the use of doped semiconductors presenting intraband or plasmonic transition in the short, mid and long-wave infrared. This strategy may offer more flexibility regarding the range of possible candidate materials. In particular, significant progress have been achieved for the synthesis of doped oxides and for the control of their doping magnitude. Among them, tin doped indium oxide (ITO) is the one providing the broadest spectral tunability. Here we test the potential of such ITO nanoparticles for photoconduction in the infrared. We demonstrate that $\ln _{2} \mathrm{O}_{3}$ nanoparticles present an intraband absorption in the mid infrared range which is transformed into a plasmonic feature as doping is introduced. We have determined the cross section associated with the plasmonic transition to be in the $1-3 \times 10^{-13} \mathrm{~cm}^{2}$ range. We have observed that the nanocrystals can be made conductive and photoconductive due to a ligand exchange using a short carboxylic acid, leading to a dark conduction with n-type character. We bring evidence that the observed photoresponse in the infrared is the result of a bolometric effect.
\end{abstract}

Keywords : Oxide nanocrystals, transport, photoconduction, plasmon, intraband absorption, infrared, heavy metal free, photodetection.

*E-mail: el@insp.upmc.fr 


\section{INTRODUCTION}

Over the recent years, huge progress ${ }^{1,2,3,4}$ have been made in the field of infrared (IR) optoelectronics using colloidal quantum dots as a low-cost alternative to historical semiconductor technologies (InGaAs, InSb, HgCdTe...). In the visible range, $\mathrm{In}^{5,6}$ has appeared as viable alternative strategy to $\mathrm{Cd}$-based nanocrystals for light emission. However, the infrared range is still driven by toxic compounds and more particularly lead $d^{7,8}$ and mercury chalcogenides ${ }^{9,10}$.

Since materials above arise a toxicological concern for mass-market applications, it is of utmost importance to screen alternative materials with a reduced toxicity and an optical absorption in the mid infrared. However, in practice narrow band gap is often found with heavy atoms which are more likely to be toxic. In this sense, doped semiconductors presenting intraband transition in the wavelength range of interest need to be considered. Mercury chalcogenides Self-doped nanocrystals ${ }^{11}$ present a tunable intraband absorption ${ }^{12,13,14}$ in the 3 to $60 \mu \mathrm{m}$ range ${ }^{15}$. $\mathrm{Ag}_{2} \mathrm{Se}^{16,17,18}$, which is heavy metal-free, presents a very similar absorption spectrum to $\mathrm{HgSe}$, but its performance for photodetection remains far weaker than its $\mathrm{Hg}$-containing counterpart ${ }^{19}$.

Degenerately doped oxides ${ }^{20,21,22}$ such as $\mathrm{Ga}^{23}, \mathrm{Al}^{24}$ and $\mathrm{In}^{25}$ doped $\mathrm{ZnO}$, indium tin oxide (ITO) ${ }^{26,27,28,29,30}$, tungsten oxide $^{31}$ and cadmium oxide have seen prompt advances in terms of synthetic maturity over the recent years. Compared with the doping of II-VI and IV-VI materials which remains difficult and mostly induced through surface effects, the doping of oxides seems more straightforward and tunable. Like bulk semiconductors, the introduction of heteroatoms leads to the generation of free carriers, resulting in plasmonic mid infrared absorption and carrier conduction. ${ }^{32,33}$ The optical absorption of such doped oxides has been extensively discussed, in particular to understand the correlation between the plasmonic absorption linewidth ${ }^{34}$ and dopant position. ${ }^{35,36,37,38}$ On the application side, such doped oxides have been driven by their use in the field of smart windows, ${ }^{39}$ sensing $^{40}$ and catalysis. ${ }^{41}$ However, their photoconductive property remains nearly unexplored. In a semiconductor, the photocurrent is proportional to two key parameters which are material absorption and photocarrier lifetime. For interband and intraband transitions, the absorption cross section per nanoparticle is quite weak, typically in the $10^{-15}$ $\mathrm{cm}^{2}$ range. ${ }^{42,43}$ This implies that for an intraband transition, from a lightly doped semiconductor (1 carrier per dot), only one electron is active every $10^{3}-10^{4}$ atoms. On the other extreme, metal with 1 active carrier per atom can reach high cross section ${ }^{44}\left(10^{-12} \mathrm{~cm}^{2}\right.$ per particle). The two types of transition also differ by the fact that intraband absorption is quantum confined (ie size dependent) while plasmonic absorption is a bulk like property. In a degenerately doped semiconductor, the situation is typically intermediate, but the cross section is higher than that of marginally doped materials. While the absorption of doped nanocrystals is expected to be large and easy to tune spectrally thanks to doping, the drawback of plasmonic absorption comes from its shorter lifetime compared with exciton which may limit the benefit of a higher absorption. Thus, the exact potential of oxide nanocrystals for IR photoconduction remains to be determined. In addition, several open questions have to be tackled such as the exact nature of the transition in the low doping regime and the potential for IR photoconduction. In addition, the electrical nature (photoconduction vs bolometric) of the response under IR illumination needs to be revealed.

In this paper, we use ITO nanocrystals as IR absorbing material because of the ease to tune its absorption spectrum thanks to doping. We then analyze the optical feature of these nanoparticles and determine their absorption cross section. We notice that the behavior of the undoped sample strongly differs from the one of the doped samples and discuss the origin of this difference. In a second step, we conduct transport measurement in a transistor configuration as a probe of the material carrier density. Finally, we have tested the potential of ITO nanocrystals as photoconductive material and compare the obtained performances with state-of-the-art IR colloidal quantum dot-containing heavy metal.

\section{DISCUSSION}

We start by synthetizing a series of ITO nanocrystals with various levels of doping from $0\left(\ln _{2} \mathrm{O}_{3}\right)$ to $15 \%$ of Sn content using a previously reported method. ${ }^{32,36} \mathrm{~A}$ scheme of the colloidally synthesized ITO nanoparticle is shown in Figure 1a. By replacing $\mathrm{In}^{3+}$ with $\mathrm{Sn}^{4+}$, free electrons are generated, so the level of doping can be tuned by changing the atomic 
ratio of $\mathrm{In}$ and $\mathrm{Sn}$ in the precursor. As can be seen in Figure $1 \mathrm{~b}$, the solution of the nanoparticles shows a color switching from colorless for undoped material to blue for doped material. The nanoparticles are of similar size ( $\approx 12 \mathrm{~nm}$ ) with a near sphere shape for all doping levels, as revealed by transmission electron microscopy (TEM), see Figure 1c and S1. The X-ray diffraction, see figure $S 2$, confirms the bixbyite structure of the $\ln _{2} \mathrm{O}_{3}$ lattice. These nanoparticles present an absorption peak in the infrared as well as a band edge in the UV range, Figure $1 \mathrm{~d}$ and S4. The inclusion of Sn, in the $\mathrm{In}_{2} \mathrm{O}_{3}$ host matrix, is confirmed by energy dispersive X-ray spectroscopy, see Figure $\mathrm{S} 5$ and table $\mathrm{S} 1$. It appears that the actual Sn content is between 50 to $70 \%$ of the introduced amount. Both transitions blueshift with the increase of Sn content. The band-edge transition shift is consistent with the bleach of the lowest energy state as the conduction band state get filled ${ }^{45}$, which is the basic principle for smart window ${ }^{46}$, see Figure 3a. The infrared peak shifts from $9.3 \mu \mathrm{m}$ ( $\approx 148 \mathrm{meV})$ for the undoped material to $2 \mu \mathrm{m}(\approx 614 \mathrm{meV})$ for the $10 \%$ doped particles, see Table 1 . We observe that above $10 \%$ the transition barely blueshifts, but strongly broadens due to an increase of electron-electron scattering ${ }^{38}$, ionized impurities scattering, alloy induced scattering and possibly due to inhomogeneous origin such as a reduced size control. Such broadening can be balanced by the introduction of $\mathrm{Cr}$ as co dopant agent ${ }^{47}$. In the following, we will focus more specifically on undoped $\left(\ln _{2} \mathrm{O}_{3}\right), 1.7 \% \mathrm{Sn}$ and $10 \% \mathrm{Sn}$ doped ITO nanoparticles.

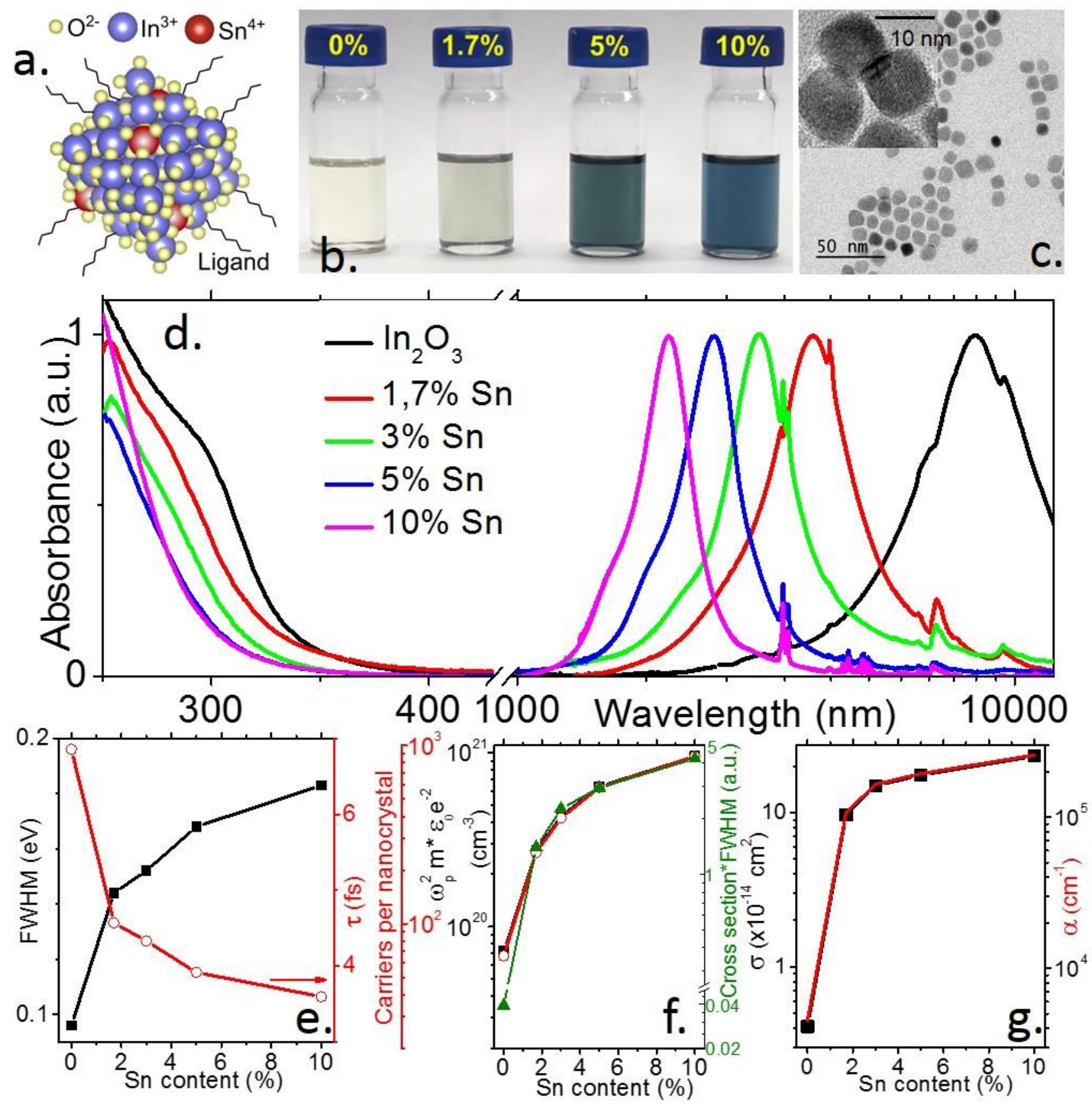

Figure 1 a. Scheme of a colloidal ITO nanoparticle with an ITO core surrounded by organic ligands. b. Image of ITO nanoparticles dispersed in $\mathrm{CHCl}_{3}$ with various levels of Sn content. c. Transmission electron microscopy image of the 
1.7\% Sn ITO nanocrystals. In the inset is the corresponding high-resolution TEM image. d. Infrared and UV-visible spectra of ITO nanocrystals with various levels of doping. e. Full width at half maximum (FWHM) of the infrared absorption and the estimated average electron scattering time $\tau(\hbar / F W H M)$ as a function of the Sn content. $f$. Estimated carrier density from the plasmon peak energy per unit of volume and per nanoparticle as a function of $\mathrm{Sn}$ content. The product of the cross section by the infrared peak FWHM is also provided. g. Absorption cross section per nanoparticle and film absorption coefficient as a function of the Sn content.

The infrared peak has been assigned to plasmonic absorption ${ }^{20,21}$, which allows us to determine the carrier density. From the Drude model ${ }^{48}$, one can relate the localized surface plasmonic resonance frequency $\left(\omega_{L S P}\right)$ to the bulk plasma frequency $\left(\omega_{p}\right)$ via $\omega_{\text {peak }}=\sqrt{\frac{\omega_{p}^{2}}{\varepsilon_{\infty}+2 \varepsilon_{M}}-\gamma^{2}}$ where $\varepsilon_{\infty}=4$ is the high frequency dielectric constant of the ITO nanoparticles, $\varepsilon_{M} \approx 2$ the dielectric constant of the environment and $\gamma$ the plasmon damping constant ${ }^{35}$. The latter is estimated as the linewidth of the plasmon transition, i.e. the full width at half maximum (FWHM) of the transition. The average scattering time $\tau$ can be estimated from $\gamma=\hbar / \tau$, with $\hbar$ the reduced Planck constant. The value of $\tau$ has been estimated to be $\approx 5 \pm 1 \mathrm{fs}$, see Figure 1 e. The plasma frequency directly relates to the carrier density $(n)$ through the relation $\omega_{p}{ }^{2}=\frac{n e^{2}}{\varepsilon_{0} m^{*}}$ with $e$ the proton charge, $\varepsilon_{0}$ the vacuum dielectric constant and $m^{*}$ the ITO conduction band effective mass, here taken equal to $0.4 \mathrm{~m}_{0}{ }^{49}, 35$ with $\mathrm{m}_{0}$ the electron rest mass.

Table 1 Band edge energy, energy of the infrared absorption peak and absorption cross section associated with the infrared transition for three Sn content of ITO nanoparticles.

\begin{tabular}{cccc}
\hline Sn content & $\begin{array}{l}\text { Band edge } \\
\text { energy (eV) }\end{array}$ & $\begin{array}{l}\text { Infrared peak } \\
\text { energy }(\mathbf{m e V})\end{array}$ & $\begin{array}{l}\text { Infrared cross } \\
\text { section }\left(\mathbf{1} \mathbf{1 0}^{-15} \mathbf{c m}^{2}\right) \\
\text { at the peak energy }\end{array}$ \\
\hline $0 \%$ & 3.8 & $148(8.3 \mu \mathrm{m})$ & 4 \\
\hline $1.7 \%$ & 4 & $315(3.95 \mu \mathrm{m})$ & 90 \\
\hline $10 \%$ & 4.3 & $614(2 \mu \mathrm{m})$ & 220 \\
\hline \hline
\end{tabular}

We estimate the carrier density to be in the $10^{20}-10^{21} \mathrm{~cm}^{-3}$ range, which corresponds to 250 free charges per $1.7 \%$ doped ITO nanoparticle and to almost $\approx 1000$ free charges in the case of the $10 \%$ doped ITO nanoparticle, see Figure 1f. In the case of the undoped material, the carrier density is estimated to be $\approx 80$ electrons per nanocrystal, however the validity of the Drude model is questionable in this case. In particular, we can notice that the product of the cross section by the IR peak linewidth follows the trend of estimated carrier density in the case of the doped sample, but is at least one order of magnitude lower for the undoped material, see Figure $1 \mathrm{f}$. Since the product of the cross section by the linewidth is an actual measurement of the effective carrier density, we can already anticipate that the Drude model largely overestimate the carrier density in the case of $\ln _{2} \mathrm{O}_{3}$.

We also have determined the absorption cross-section of the nanoparticles as a function of doping, see Figure $1 \mathrm{~g}$, Table 1 and Methods. Doped nanoparticles have a cross section, at the infrared peak energy, in the $1-3 \times 10^{-13} \mathrm{~cm}^{2}$ range $e^{50}$. This corresponds to absorption coefficient in the $1 \times 10^{5}$ to $3 \times 10^{5} \mathrm{~cm}^{-1}$. While the cross section increases quasi linearly with doping for doped nanoparticles, the undoped nanoparticles present a much weaker absorption with a cross section of only $4 \times 10^{-15} \mathrm{~cm}^{2}$.

Transport measurements are then used to probe the carrier density. To ensure a strong interparticle coupling in the film, a ligand exchange step is required. While thiols have been extensively used for $\mathrm{Cd}, \mathrm{Hg}$ or $\mathrm{Pb}$ based nanocrystals, the hard base nature of $\mathrm{In}^{3+}$ leads to a poor affinity for thiol. As a result, we choose a hard acid ${ }^{51}$, according to Pearson's theory, such as acetic acid to replace the initial oleic acid ligands ${ }^{26}$. The transport properties of the ITO nanocrystals under dark condition are probed using an electrolyte field effect transistor configuration, see a scheme of the setup in Figure 2a. Electrolyte gating, here made by dissolving $\mathrm{LiClO}_{4}$ in a polyethylene glycol matrix, ensures a large gate 
capacitance which is critical to modulate the carrier density of degenerately doped material. In addition, it allows air operation and gating of thick films ${ }^{52}$.
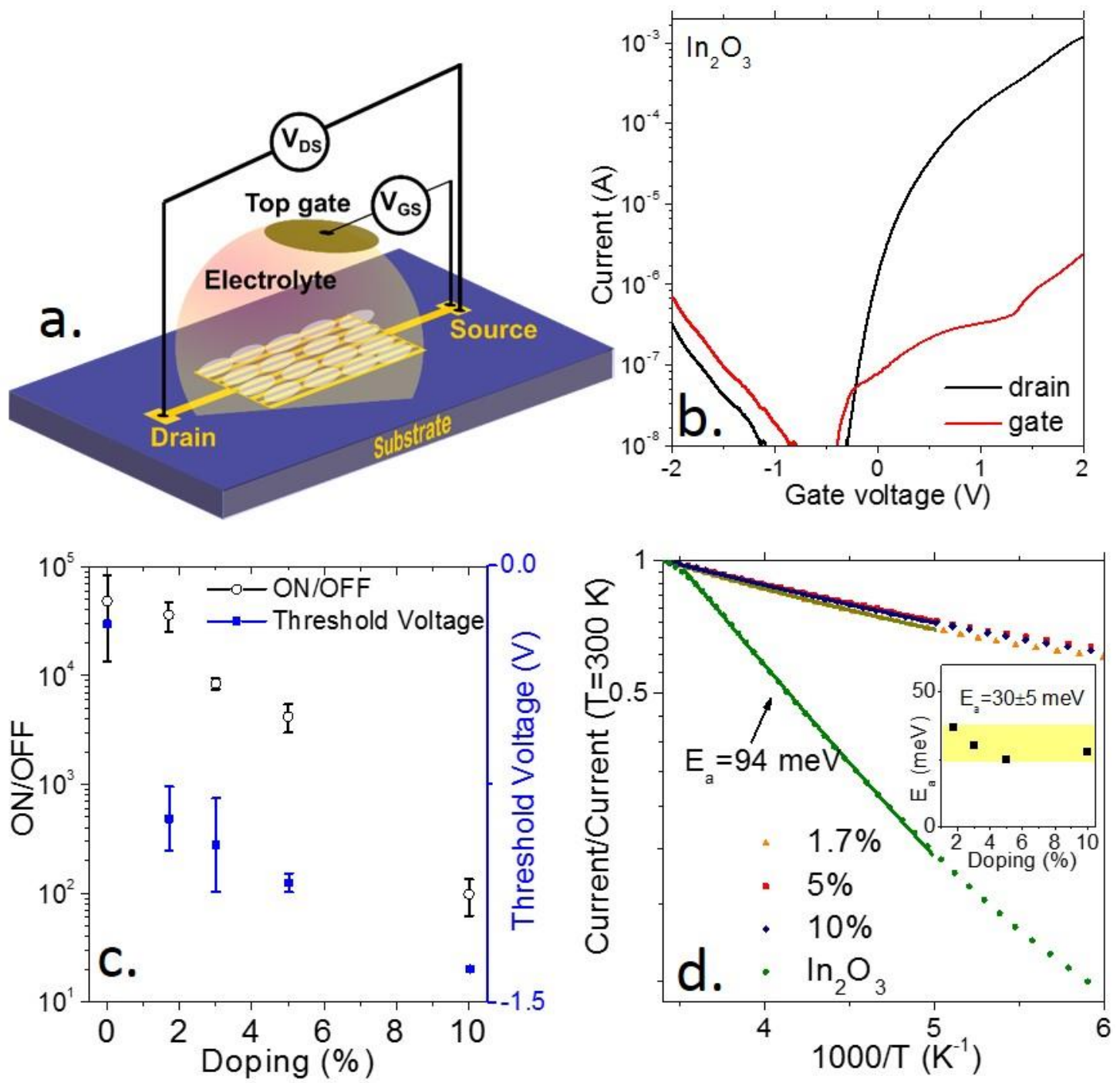

Figure 2a. Scheme of an electrolyte gated transistor with a thin film of ITO nanocrystals as canal. $b$. Transfer curve (drain and gate current as a function of the applied gate voltage) for a transistor whose canal is made of $\ln _{2} \mathrm{O}_{3}$ nanocrystals. c. On/off ratio and threshold voltage as a function of the Sn content for a transistor made of ITO nanocrystals thin film. $d$. Current as a function of temperature for ITO nanocrystal thin films with various Sn contents. The inset provides the fitted activation energy for the doped samples assuming an Arrhenius fit of the high temperature part of the I-T curve.

Thin films of ITO and undoped $\ln _{2} \mathrm{O}_{3}$ nanoparticles, where transport properties are probed in a field effect transistor configuration ${ }^{52}$, only present $n$-type conduction (i.e. rise of conduction under positive gate bias), see Figure $2 b$ and Figure S9. No evidence for hole transport has been observed, which is consistent with previous reported hall effect ${ }^{53}$ and thermoelectric measurements ${ }^{22}$. We nevertheless observe a clear reduction of the current modulation (on/off ratio) as well as a shift of the threshold voltage toward more negative potentials as the Sn content increases, see Figure 2c. Both are consistent with the increase of doping leading to a Fermi level deeper within the conduction band. Around room temperature, the transport is thermally activated, see Figure $2 \mathrm{~d}$. An activation energy of almost $100 \mathrm{meV}$ in the case of $\mathrm{In}_{2} \mathrm{O}_{3}$ is extracted from the Arrhenius fit of the I-T curve, while for doped samples the value is $30 \pm 5 \mathrm{meV}$, independent of the doping level, see the inset of Figure $2 \mathrm{~d}$.

Hopping transport ${ }^{33,54,55}$ is typically characterized by different behaviors between semiconductor and metal. In semiconductor material, hopping is generally described using a Mott model, which indicates that the transfer of carriers from one particle to another is limited by the availability of empty arrival state. Thus, the activation energy reflects the density of states of the nanoparticle ${ }^{56,57}$. If the states are sparse, fairly large value of activation energy can 
be obtained, as is the case for undoped $\ln _{2} \mathrm{O}_{3}$ nanoparticles. On the other hand, for metal and strongly doped nanoparticles, the density of states is no longer limiting and only the coulombic charging energy $\left(\mathrm{E}_{\mathrm{c}}\right)$ has to be overcome ${ }^{58}$. The latter value can be evaluated using $E_{C}=\frac{e^{2}}{2 C},{ }^{58}$ with $C$ the self-capacitance of the nanoparticle. $C$ can be estimated from $C=2 \pi \varepsilon_{0} \varepsilon_{r} d$, with $\varepsilon_{0}$ the vacuum permittivity, $\varepsilon_{r}$ the material dielectric constant (4) and $d$ the nanoparticle diameter ( $12 \mathrm{~nm}$ ), which leads to a $E_{\mathrm{c}}$ of $29 \mathrm{meV}$ for ITO nanoparticles. In this sense, the characteristic energy of the hopping transport in the array of degenerately doped semiconductors is the same one as for metallic nanoparticles. ${ }^{58}$

While dark conduction in ITO nanoparticle film has already been explored, ${ }^{32,33,59}$ their photoconductive properties remain mostly unexplored. We choose to study their photoconductive properties by exciting them at the band edge (UV irradiation) or by directly exciting their plasmonic feature in the near-IR for $10 \%$ doped and in the mid-IR for the $1.7 \%$ doped nanoparticles. In practice, we use four light sources to resonantly excite each of these transitions, see the simplified band diagram in Figure 3a. The three levels of doping actually corresponds to atmospheric transparency windows where long distance imaging is possible. The $10 \%$ doped material overlaps with the short wave infrared (SWIR: $1-3 \mu \mathrm{m}$ ), the $1.7 \%$ doped material is in the mid wave infrared (MWIR: $3-5 \mu \mathrm{m}$ range) and the undoped material is in the long wave infrared range (LWIR: 8-12 $\mu \mathrm{m}$ ).
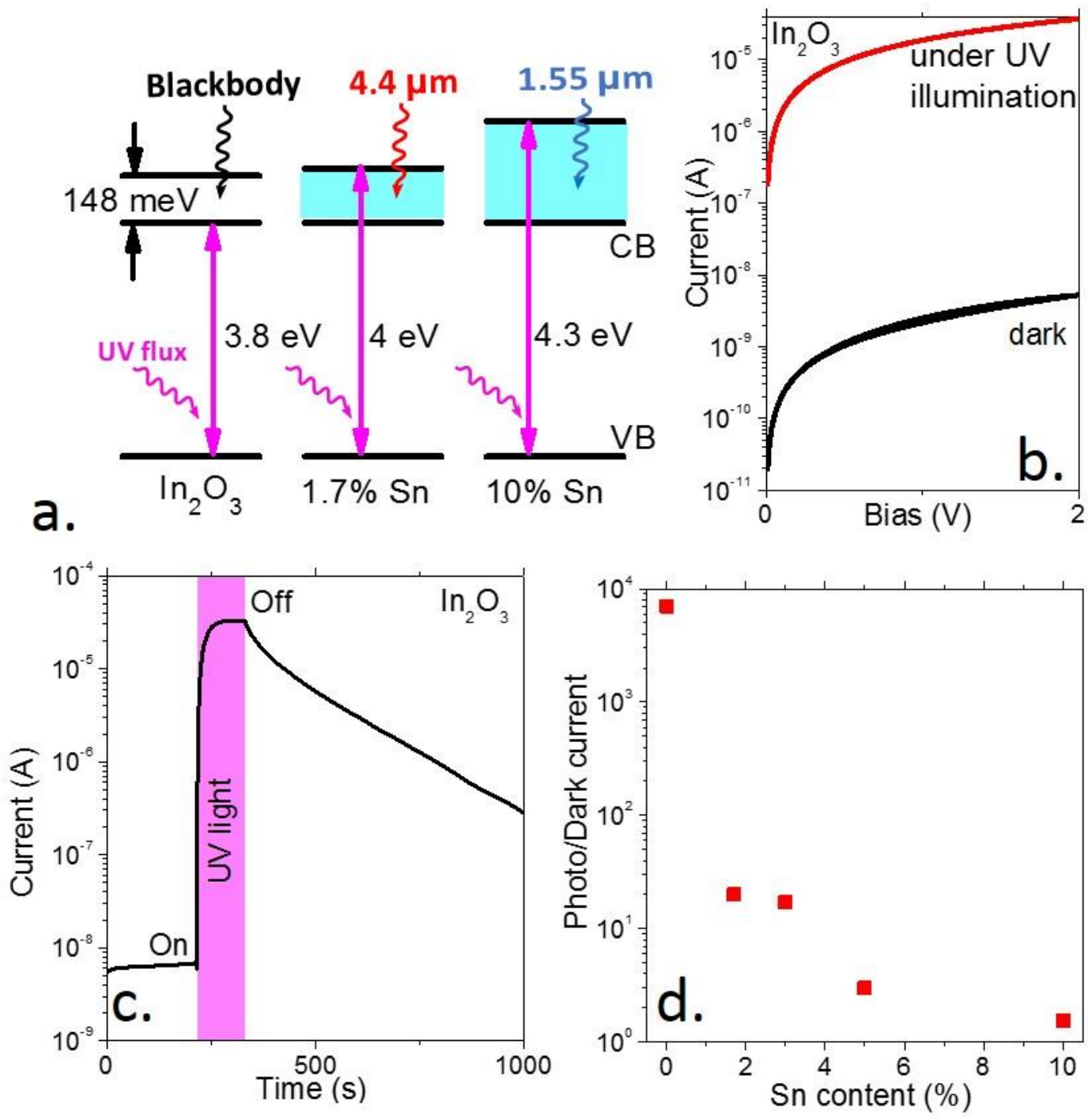

Figure $3 a$. Band diagram of undoped, $1.7 \%$ Sn and $10 \%$ Sn doped ITO nanocrystals. For all materials, the bottom of the conduction band and the top of the valence band are treated as identical to the undoped material. The energy of the fisrt empty state of the conduction band is determined using a Tauc plot, see figure S3. b. I-V curve of $\operatorname{In}_{2} \mathrm{O}_{3}$ nanoparticles under dark condition and under UV illumination. c. Current as a function of time while a thin film $\operatorname{In}_{2} \mathrm{O}_{3}$ 
nanoparticles is exposed to a pulse of UV light. $d$. Current modulation (ratio of the photocurrent over the dark current) as function of the Sn content of an ITO thin film exposed to a short illumination of UV light.

Under UV excitation, a large current modulation is observed, see Figure 3 and S10. A striking feature relative to the excitation of the band edge is the slow response of the photocurrent, see Figure $3 \mathrm{c}$. The decay time can be as long as $1 \mathrm{~h}$, which is the signature of the involvement of deep traps in the photoconductive process ${ }^{60}$. Similar memory effect has already been observed for $\mathrm{ZnO}^{61,62}$ nanoparticles and are now used as a strategy to activate the conduction of electron transport layer ${ }^{63}$ in a solar cell device. The magnitude of the photoresponse tends to be reduced while the $\mathrm{Sn}$ content is increased. Again, the undoped nanoparticles present a significantly larger modulation than all doped nanoparticles, see Figure $3 d$, due to a much lower dark current. This observation combined with the weaker cross section of this material confirms that the free carrier density in the undoped material is low and certainly much weaker than the one determined using a Drude model. This suggests that the infrared peak observed for $\ln _{2} \mathrm{O}_{3}$ nanoparticles has an intraband character, rather than plasmonic. In the case of ITO, the transition between these two regimes occurs for a carrier density below 100 electrons / particle $\left(\approx 10^{20} \mathrm{~cm}^{-3}\right)$. Once the doping is introduced, this transition acquires a more collective character as it has been proposed in the case of self-doped HgS quantum dots ${ }^{64}$ and doped $\mathrm{ZnO}$ nanocrystals ${ }^{65}$. The transition is well modeled using a quantum plasmon model, which converge toward the electron transition, in the low carrier density limit. ${ }^{65}$

To test the plasmonic character of this transition, we have tried, as proposed by the Milliron's group ${ }^{29}$ to change the solvent to tune the dielectric constant of the medium. We speculate that plasmonic transition will be more sensitive to such shift than intraband absorption. However this experiment has been unsucesfull for $\ln _{2} \mathrm{O}_{3}$ due to the strong absorption of solvent, even chlorinated ones, around $1000 \mathrm{~cm}^{-1}$, see figure $\mathrm{S} 6$. We thus choose to use another approach to support this claim, we graft polyoxometalates (POM) on the surface of the nanoparticles. It was recently demonstrated by Martinez et $a{ }^{66}$ that POM can be used as agents to tune the doping magnitude of HgSe nanocrystals. The POMs used contain tungsten atoms under a strongly oxidized (+VI) form, which can behave as electron attractors. Once POMs are grafted on the surface of degenerately $n$-doped nanoparticles, they can strip electrons from the nanoparticles, which tends to reduce the oxidation state of the tungsten from the POM as evidenced by photoemission in ref 66. For doped forms of ITO, the grafting of the POMs has a severe effect on the linewidth of the plasmonic transition, see Figure $4 \mathrm{~b}$ and $\mathrm{S} 8$. This is at first a counter intuitive result since a reduction of the electron density may have been expected to lead to a redshift of the plasmonic transition. This scenario can be explained that the POMs rather disturb the radial distribution of carrier, as depicted in Figure 4a. Initially ITO behaves as a Jellium ${ }^{38}$, meaning that positive charges resulted from Sn dopants are fixed while free electrons are homogeneously spread all over the nanoparticle. After the POM grafting, the nanoparticle surface is electron deficient, which leaves positive impurities unscreened on the surface. This tends to enhance the electron impurity scattering and leads to the observed increase of the spectrum linewidth. ${ }^{38}$

On the other hand, for $\operatorname{In}_{2} \mathrm{O}_{3}$, the infrared peak is neither shifted nor broaden, see Figure $4 \mathrm{c}$. In other words, the IR spectrum of the $\operatorname{In}_{2} \mathrm{O}_{3}$ nanoparticles with grafted POMs is just the sum of the absorption of the two materials. This reflects the fact that this transition is determined by the density of states of the semiconductor, rather than the electron gas distribution. This further supports the intraband character of the transition in $\ln _{2} \mathrm{O}_{3}$. 

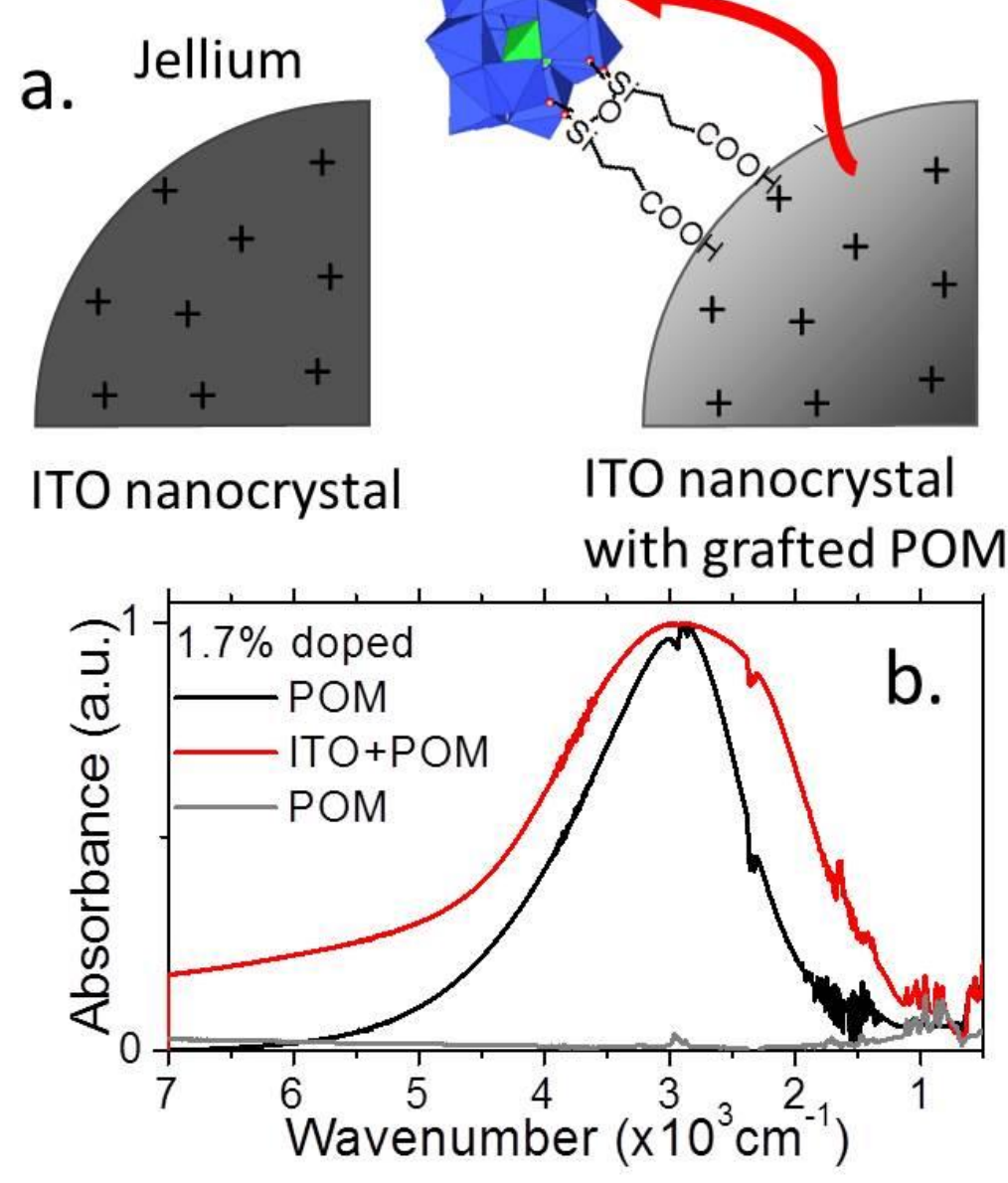

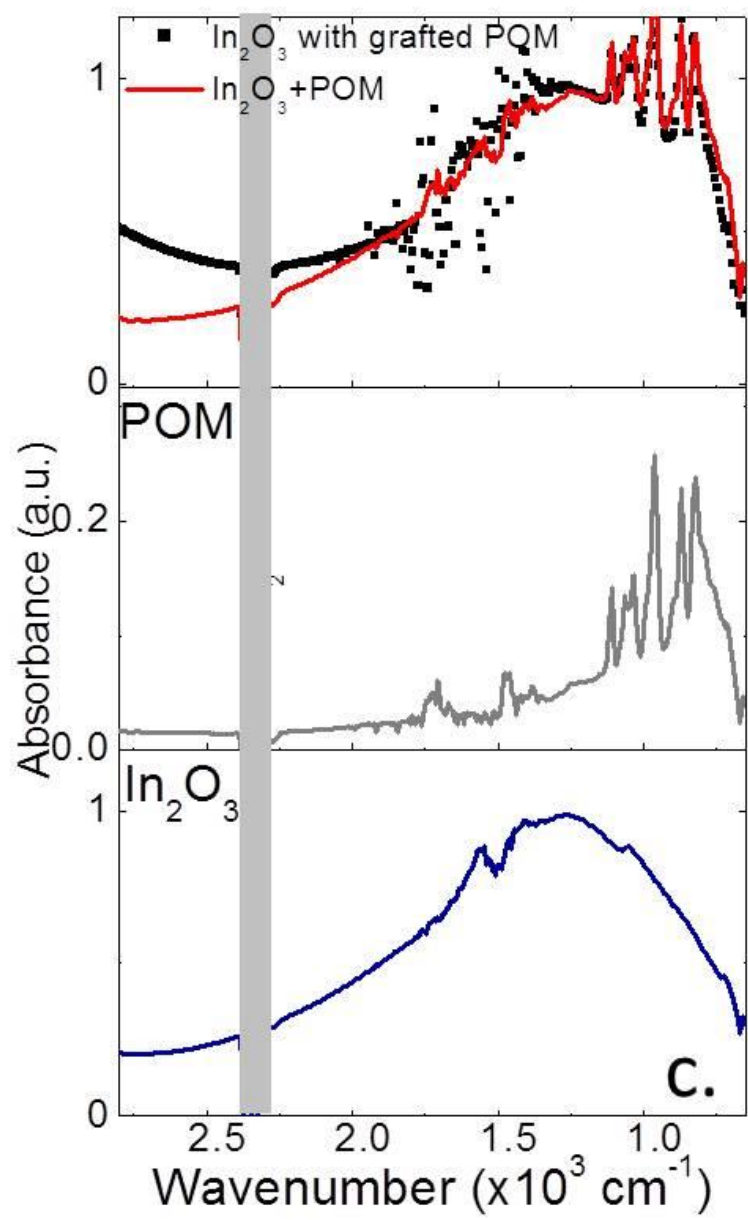

Figure 4 a. Scheme of a non-functionalized nanoparticle where positive charges coming from Sn dopants are randomly dispersed in the nanocrystal, leading to a homogeneous distribution for the electron gas (in grey). In presence of electron attracting groups at the nanoparticle surface (here polyoxometalates), surface is electronically depleted and leave some of the positive charges unscreened. $b$. Infrared spectra of $1.7 \%$ doped ITO with and without POM grafted on the surface. c. Infrared spectrum of the $\operatorname{In}_{2} \mathrm{O}_{3}$ (bottom part), pristine POM (central part) and from the $\operatorname{In}_{2} \mathrm{O}_{3}$ nanoparticles grafted with POM (top part, scatter). The latter signal is well fitted (red curve) by an addition of the signal from the independent POM and $\ln _{2} \mathrm{O}_{3}$ nanoparticles.

There are several significant consequences to this observation beyond the obvious facts that undoped nanoparticles have lower conductivity and weaker absorption. Because the transition in $\ln _{2} \mathrm{O}_{3}$ is determined by the semiconductor density of states, the energy of this transition will be only marginally tunable through doping. Reducing the doping and even the residual material non-stoichiometry will not allow to redshift the transition of ITO toward wavelengths longer than $9 \mu \mathrm{m}$. This may be a limitation for detection application in the low energy part of the 8-12 $\mu \mathrm{m}$ atmospheric transparency window. Secondly, if tuning of the intraband transition of $\operatorname{In}_{2} \mathrm{O}_{3}$ needs to be investigated, alternative paths such as change of confinement or material alloying need to be considered.

In the last part of the paper, we aim to discuss the potential of ITO nanocrystals as photoconductive material in the infrared range. Three ranges of detection have been considered : long-wave infrared for $\ln _{2} \mathrm{O}_{3}$ nanoparticles, midwave infrared for $1.7 \%$ doped ITO and short-wave infrared for $10 \%$ doped ITO, see Figure 3a. Samples are cooled down to reduce their thermally activated carrier density, see Figure $5 \mathrm{a}, \mathrm{d}$ and $\mathrm{g}$. The responsivity achieved by the film of $\mathrm{In}_{2} \mathrm{O}_{3}$ nanocrystals reaches $40 \mu \mathrm{A} / \mathrm{W}$ in the long wavelength range, $4 \mu \mathrm{A} / \mathrm{W}$ in the mid infrared (1.7\% doped ITO at 4.4 $\mu \mathrm{m})$ and $14 \mu \mathrm{A} / \mathrm{W}$ at $1.55 \mu \mathrm{m}$ for the $10 \% \mathrm{Sn}$ ITO. These performances are weak compared with the one reported for HgTe nanocrystals with interband transition at the same wavelength, in which case responsivity in the 1 to $100 \mathrm{~mA} / \mathrm{W}$ 
is currently achieved ${ }^{2,4}$. This is, on the other hand, similar to other $\mathrm{Hg}$-free material such as $\mathrm{Ag}_{2} \mathrm{Se}^{19}$ nanocrystals, which present intraband absorption around $5 \mu \mathrm{m}$ and for which responsivity reaches $8 \mu \mathrm{A} / \mathrm{W}$.

The $\ln _{2} \mathrm{O}_{3}$ nanoparticles present a dramatically slow photoresponse with turn-on and turn-off time close to $10 \mathrm{~min}$, Figure $5 \mathrm{~b}$. All doped nanoparticles have a similar time response, with a turn-on time above $10 \mathrm{~s}$ and a turn-off time of $\approx 1$ min typically, see Figure $5 \mathrm{e}$ and $\mathrm{h}$. Even if their dynamics are shorter than the photoconductive dynamics at the band edge, they remain extremely slow compared with other IR active colloidal materials in the same range of wavelengths. For example, with HgTe quantum dots, time response shorter than the $\mu$ s are commonly reported. ${ }^{67,68}$ This long-time response suggests that the current modulation results from a bolometric effect. In other words, the change of carrier density induced by the light (i.e. the photocurrent) is negligible compared to the increase of thermally activated carrier density (i.e. the light-induced change of the dark current).

This result may have been anticipated from the evaluation of the cross section $(\sigma)$ and photocarrier lifetime $(\tau)$. Indeed we can estimate the photogenerated change of carrier density under illumination to be ${ }^{69} \Delta n=e \sigma \tau \Phi / E_{p h}$ with $e$ the proton charge, $\Phi$ the power flux per unit area and $E_{p h}$ the photon energy. In the case of the $1.7 \%$ doped ITO nanoparticle $\left(\sigma \approx 10^{-13} \mathrm{~cm}^{2} ; \tau \approx 5 \mathrm{fs}\right)$, with the illumination condition used in Figure $5 \mathrm{e}\left(E_{p h}=0.28 \mathrm{eV}\right)$, the light induced change of carrier density is $\approx 5 \times 10^{-9}$ electron/particle, which has to be compared with the steady doping of 200 electron /particle. As the photocurrent and dark current is respectively proportional to these two quantities, the ratio of photo to dark current is thus expected to be very small. Experimentally, the light to dark modulation is nevertheless higher (in the $10^{-3}$ to 10 range) than this ratio, see Figure $5 c, f$ and $i$, which confirms that the bolometric origin is prevailing.

We can conclude that the benefit of a stronger absorption brought by the plasmonic absorption is strongly balanced by the short lifetime of the photocarrier. Thus, to take full benefit of the larger plasmonic absorption, a fast extraction of the hot electrons will have to be implemented. A possible strategy to reach this goal, will be to reduce the device size down to the $\mathrm{nm}$ scale, which will be valuable to apply large electric field and reduce the carrier transit time. ${ }^{70}$ Regarding the operating temperature of such films, we measured that the photocurrent remains a marginal modulation compared with the dark current, see Figure $5 \mathrm{c}$, $\mathrm{f}$ and i. Only the undoped material achieve BLIP (background limited performances, defined here as the temperature when dark current becomes smaller than the photocurrent) operation at $\mathrm{T}<50 \mathrm{~K}$, see Figure $5 \mathrm{c}$. This is the result of the large doping, required to achieve plasmonic absorption, but which comes at the price of a large dark current. 

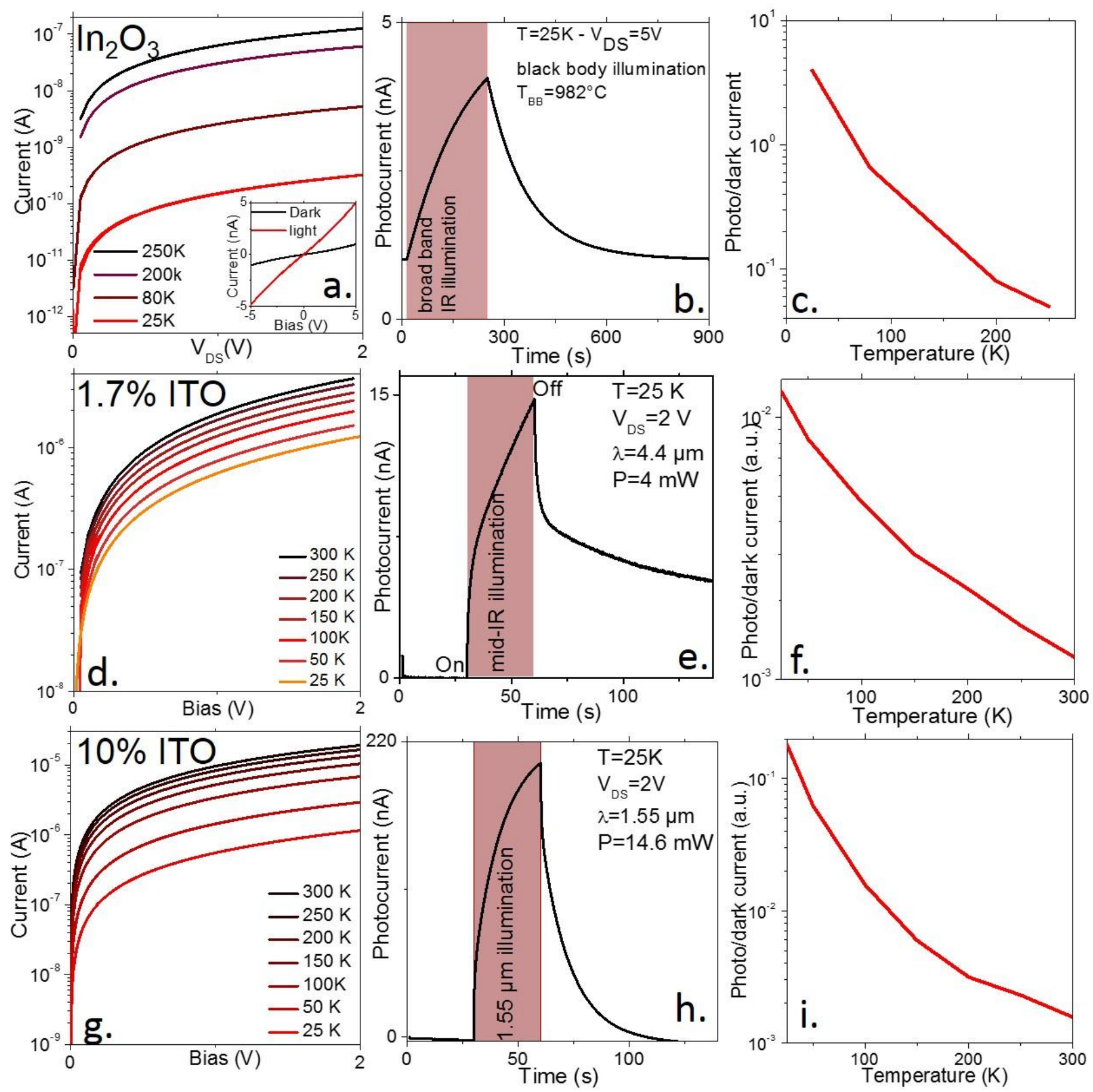

Figure $5 \mathrm{I}-\mathrm{V}$ curves for a thin film of (a) $\mathrm{In}_{2} \mathrm{O}_{3}$ nanoparticles, (d) 1.7\% doped ITO nanoparticles and (g) $10 \%$ doped ITO nanoparticles at various temperatures ranging from room temperature down to $25 \mathrm{~K}$. The inset in $(a)$ is the I-V curves at $25 \mathrm{~K}$ under dark condition and under illumination by a blackbody source $\left(T_{B B}=982^{\circ} \mathrm{C}\right)$. Current as a function of time of a thin film of (b) $\ln _{2} \mathrm{O}_{3}$ nanoparticles, (e) $1.7 \%$ doped ITO nanoparticles and (h) $10 \%$ doped ITO nanoparticles while exposed to (b) a pulse illumination by a blackbody source, (e) a short illumination of a quantum cascade laser operating at $4.4 \mu \mathrm{m}$ and (h) a short illumination resulting from $1.55 \mu \mathrm{m}$ laser diode. Ratio of the photocurrent over the dark current as a function of the temperature for a thin film of (c) $\ln _{2} \mathrm{O}_{3}$ nanoparticles exposed to the excitation from a blackbody source, (f) 1.7\% doped ITO nanoparticles exposed to the excitation from a $4.4 \mu \mathrm{m}$ QCL and (i) $10 \%$ doped ITO nanoparticles exposed to the excitation from a $1.55 \mu \mathrm{m}$ laser diode.

\section{CONCLUSION}

To summarize, we have tested the potential of ITO nanocrystals for heavy metal free infrared photoconduction. We have determined that the undoped $\ln _{2} \mathrm{O}_{3}$ nanoparticles present an intraband absorption at 8-9 $\mu \mathrm{m}$ which transforms into a plasmonic transition as the $\mathrm{Sn}$ doping is introduced. The intraband character of this transition will make the tuning of the absorption toward longer wavelength more challenging. Doped nanoparticles of ITO present a cross 
section of a few $10^{-13} \mathrm{~cm}^{2}$ per nanoparticle, corresponding to an absorption coefficient above $10^{5} \mathrm{~cm}^{-1}$. The material presents n-type conduction with a weak temperature dependence. Photoconduction in the infrared range can be obtained at low temperature and results from a bolometric effect. The responsivity achieved by thin films of ITO nanoparticle are in the few to few tens of $\mu \mathrm{A} / \mathrm{W}^{-1}$ range. This makes the obtained photosignal weak compared with the dark current, as a result, ITO nanoparticle are not yet competitive with other materials such as PbS and HgTe. Future development will have to integrate a strategy to take advantage of the short living hot electrons.

\section{METHODS}

\section{List of chemicals}

Indium(III) acetate (Sigma-Aldrich, $99.99 \%$ ), tin(IV) acetate ( Alfa Aesar), oleic acid (Sigma-Aldrich), olyel alcohol (Alfa Aesar, tech. 80-85\%), ethanol absolute anhydrous (Carlo Erba, 99.9\%), Chloroform (Carlo Erba), n-hexane (Carlo Erba), n-octane (SDS, 99\%), acetic acid (Sigma-Aldrich, $\geqslant 99 \%), \mathrm{N}, \mathrm{N}$-dimethylformamide (DMF, Sigma Aldrich), triethyloxonium tetrafluoroborate ( $\mathrm{Et}_{3} \mathrm{O}^{+} \mathrm{BF} 4$, Sigma Aldrich), lithium perchlorate $\left(\mathrm{LiClO}_{4}\right.$, Sigma-Aldrich, 98\%), polyethylene glycol (PEG, Mw=6 kg/mol) were used as received.

Synthesis of indium oxide and Sn-doped indium oxide nanocrystals: In this study, the nanocrystals were synthesized with a two-step slow-injection method established by Jansons et al. ${ }^{36}$ with minor modifications. In one three-neck flask, a desired composition of indium acetate and tin (IV) acetate ( $5 \mathrm{mmol}$ in total) was mixed with oleic acid (10 $\mathrm{mL}$ ). The mixture was heated at $150{ }^{\circ} \mathrm{C}$ under Ar until all the powders are fully dissolved (typically 1 hour). The obtained metal oleate solution (0.5M) was light yellow in color. In another flask, $13 \mathrm{~mL}$ of oleyl alcohol was heated to $305^{\circ} \mathrm{C}$ under Ar. Then $1 \mathrm{~mL}$ of the as-prepared metal oleate solution was injected to the oleyl alcohol bath using a syringepump at a rate of $0.2 \mathrm{mg} / \mathrm{mL}$. After the injection, the reaction was baked at $305^{\circ} \mathrm{C}$ under Ar for 20 min before cooled down with air flux. The obtained nanocrystals were precipitated with ethanol and redispersed in chloroform for 3 times and finally dispersed in chloroform for storage. The nominal doping of $\mathrm{Sn}$ in nanocrystal was determined by the $\mathrm{Sn} /(\mathrm{In}+\mathrm{Sn})$ ratio in the mixed precursor. In this manner, we synthesized a series of nanocrystals with $0 \%\left(\ln _{2} \mathrm{O}_{3}\right), 1.7 \%$, $3 \%, 5 \%$ and $10 \%$ of Sn doping.

POM-COOH (TBA) $)_{3}\left[\mathrm{PW}_{11} \mathrm{O}_{39}\left\{\mathrm{O}\left(\mathrm{SiC}_{2} \mathrm{H}_{4} \mathrm{COOH}\right)_{2}\right\}\right]$ synthesis ${ }^{71}: \mathrm{K}_{7}\left[\mathrm{PW}_{11} \mathrm{O}_{39}\right](0.64 \mathrm{~g}, 0.2 \mathrm{mmol})$ was dissolved in a water/acetonitrile mixture $(30 \mathrm{~mL}, 1: 2)$. A $1 \mathrm{M} \mathrm{HCl}$ aqueous solution was added drop by drop until an apparent $\mathrm{pH}$ equals to 3. The solution was cooled in an ice bath and the $\mathrm{Si}(\mathrm{OH})_{3}\left(\mathrm{CH}_{2}\right)_{2} \mathrm{COONa}(0.476 \mathrm{~mL}, 0.8 \mathrm{mmol})$ was inserted. The $1 \mathrm{M} \mathrm{HCl}$ solution was added drop by drop again to reach $\mathrm{pH}_{\mathrm{app}}=2$. After an overnight reaction, $\mathrm{TBABr}(0.26 \mathrm{~g}, 0.8$ $\mathrm{mmol}$ ) was added and the solution concentrated with a rotary evaporator to make precipitate the product. The oily compound obtained was dissolved in the minimum of acetonitrile then precipitated again with an excess of ether. A sticky solid was recovered by centrifugation and washed thoroughly with ether to obtain a white powder ( $0.6 \mathrm{~g}, 82 \%$ ). The dried compound was found to be partially deprotonated (cf. IR and EA analysis) with the exact general formula $(\mathrm{TBA})_{3,4}\left[\mathrm{PW}_{11} \mathrm{O}_{39}\left\{\mathrm{O}\left(\mathrm{SiC}_{2} \mathrm{H}_{4} \mathrm{COOH}_{0,8}\right)_{2}\right\}\right]$.

${ }^{1} \mathrm{H}$ NMR $\left(400 \mathrm{MHz}, \mathrm{CD}_{3} \mathrm{CN}\right): \delta(\mathrm{ppm}) 3.14(\mathrm{~m}, 24 \mathrm{H}), 2.53(\mathrm{~m}, 4 \mathrm{H}), 1.65(\mathrm{~m}, 24 \mathrm{H}), 1.41\left(\mathrm{sex},{ }^{3} \mathrm{~J}(\mathrm{H}, \mathrm{H})=7.5 \mathrm{~Hz}, 24 \mathrm{H}\right), 1.05$ $(\mathrm{m}, 4 \mathrm{H}), 1.01\left(\mathrm{t},{ }^{3} \mathrm{~J}(\mathrm{H}, \mathrm{H})=7.5 \mathrm{~Hz}, 36 \mathrm{H}\right), 0.90(\mathrm{~m}, 4 \mathrm{H})$;

${ }^{31} \mathrm{P}$ NMR $\left(121 \mathrm{MHz}, \mathrm{CD}_{3} \mathrm{CN}\right) \delta(\mathrm{ppm})-12.28$;

IR (KBr pellet) : $\delta=2963$ (s), 2935 (m), $2874(w), 1710(\mathrm{~s}), 1623$ (w), 1483 (s), 1471 (s), 1420 (w), 1381 (m), 1112 (vs), 1064 (vs), 1052 (s), 1036 (s), 964 (vs), 870 (vs), 824 (vs) 
MS (ESI-), $\mathrm{m} / \mathrm{z}$ (\%) : calcd for $\mathrm{W}_{11} \mathrm{PSi}_{2} \mathrm{O}_{44} \mathrm{C}_{6} \mathrm{H}_{10}: 965.41[\mathrm{M}]^{3-}$; found : 965.42 (100) ; calcd for $\mathrm{W}_{11} \mathrm{PSi}_{2} \mathrm{O}_{44} \mathrm{C}_{22} \mathrm{H}_{46} \mathrm{~N}: 1569.26$ $[\mathrm{M}+\mathrm{TBA}]^{2-} ;$ found : $1569.27(50)$

Elemental Analysis calcd (\%) for $\mathrm{C}_{60.4} \mathrm{H}_{132} \mathrm{~N}_{3.4} \mathrm{O}_{44} \mathrm{Si}_{2} \mathrm{PW}_{11}$ : C 19.50, H 3.55, N 1.28; found : C 19.51, H 3.50, N 1.24

POM grafting on ITO nanoparticles: We used a two-step ligand exchange method with the removal of organic ligands followed by the functionalization with POM. Firstly $1 \mathrm{~mL}$ of NCs in hexane $(10 \mathrm{mg} / \mathrm{mL})$ was mixed with $1 \mathrm{~mL}$ of triethyloxonium tetrafluoroborate in DMF $(20 \mathrm{mg} / \mathrm{mL})$ vigorously with vortex and sonication. After $5 \mathrm{~min}$, the NCs were transferred from nonpolar phase (hexane) to the polar one (DMF) on the bottom, due to the stripping of their organic ligands. Then, the bare NCs in DMF were precipitated with toluene and redissolved in $1 \mathrm{~mL}$ of POM in DMF (20 $\mathrm{mg} / \mathrm{mL}$ ) for functionalization. After complete mixing, the POM-capped NCs were precipitated with toluene to remove the POM excess and were redispersed in DMF.

Ligand exchange for transport measurements: To improve electron transport, the long organic ligands of nanocrystals were replaced by short ones using an on-film ligand exchange method. In more details, we first drop-casted the nanocrystal solution onto a substrate. Once the substrate was dried, it was dipped into a solution of acetic acid in ethanol ( $0.5 \mathrm{wt} \%$ ) for $60 \mathrm{~s}$ then in pure ethanol for $30 \mathrm{~s}$. The substrate was then annealed at $250^{\circ} \mathrm{C}$ for $15 \mathrm{~min}$ to finish one round of ligand exchange. The procedure was repeated once before transport measurements.

Material characterization: Absorption spectra are acquired using a Jasco V730 spectrometer for the UV visible part, while a Thermo Fischer IS50 Fourrier transform infrared spectrometer in ATR configuration is used in the IR range. For the determination of the absorption cross section, a film of nanoparticles has been deposited onto a double side polished Si wafer. Its thickness is determined using a Dektak 150 profilometer. The absorption coefficient is given by $\frac{A \cdot \ln 10}{t \cdot f}$ with $\mathrm{A}$ the absorption, $\mathrm{t}$ the film thickness, and $\mathrm{f}$ the film volume fraction taken equal to 0.64 which correspond to a randomly close pack film. The cross section per particle is simply obtained by multiplying the absorption coefficient by the nanoparticle volume, assuming a spherical shape. For transmission electron microscopy, we used a JEOL 2010. The grids were prepared by a drop cast of dilute solution of nanocrystals dispersed in hexane and degassed overnight under secondary vacuum. X-ray diffraction pattern is obtained by drop casting a solution of nanocrystals on a Si wafer. The diffractometer is a Philips X'Pert, based on the emission of the Cu Ka line operated at $40 \mathrm{kV}$ and $40 \mathrm{~mA}$ current.

$\mathrm{Si} / \mathrm{SiO}_{2}$ substrate for electrodes: The surface of a $\mathrm{Si} / \mathrm{SiO}_{2}$ wafer ( $400 \mathrm{~nm}$ oxide layer) is cleaned by sonication in acetone. The wafer is rinsed with isopropanol and finally cleaned using an $\mathrm{O}_{2}$ plasma. AZ 5214E resist is spin-coated and baked at $110^{\circ} \mathrm{C}$ for $90 \mathrm{~s}$. The substrate is exposed under UV through a pattern mask for $2 \mathrm{~s}$. The film is further baked at $125^{\circ} \mathrm{C}$ for $2 \mathrm{~min}$ to invert the resist. Then a $40 \mathrm{~s}$ flood exposure is performed. The resist is developed using a bath of AZ 326 for $32 \mathrm{~s}$, before being rinsed in pure water. We then deposit a $5 \mathrm{~nm}$ chromium layer and $80 \mathrm{~nm}$ gold layer using a thermal evaporator. The lift-off is performed by dipping the film in acetone for $1 \mathrm{~h}$. The electrodes are finally rinsed using isopropanol and dried by an air flow. The electrodes are $2.5 \mathrm{~mm}$ long and spaced by $20 \mu \mathrm{m}$. These electrodes are used for DC measurements (IV curves and transistor measurements).

Electrolyte gating: For electrolyte gating, we first mix in a glove box $0.5 \mathrm{~g}$ of $\mathrm{LiClO}_{4}$ with $2.3 \mathrm{~g}$ of PEG $\left(\mathrm{MW}_{\mathrm{w}}=6 \mathrm{~kg} \cdot \mathrm{mol}^{-}\right.$ $\left.{ }^{1}\right)$. The vial is heated at $170^{\circ} \mathrm{C}$ on a hot plate for $2 \mathrm{~h}$ until the solution gets clear. To use the electrolyte, the solution is warmed around $100^{\circ} \mathrm{C}$ and brushed on the top of the ITO nanoparticle film. 


\section{Electrical measurements}

DC transport: The sample is connected to a Keithley 2634b which applied bias and measured current. For measurement under illumination three sources has been used: a UV flash light at $365 \mathrm{~nm}$, a $1.55 \mu \mathrm{m}$ laser diode and a quantum cascade laser operating at $4.4 \mu \mathrm{m}$. For measurement as a function of temperature, the sample is mounted on the cold finger of a cryostat and the sample is biased using a Keithley 2634b. The current is acquired while the temperature of the sample is cooled down.

Transistor measurements: The sample is connected to a Keithley $2634 \mathrm{~b}$ which sets the drain bias $\left(\mathrm{V}_{\mathrm{DS}}=20 \mathrm{mV}\right.$ for doped ITO, $V_{D S}=200 \mathrm{mV}$ for $\left(\mathrm{n}_{2} \mathrm{O}_{3}\right)$, controls the gate bias $\left(\mathrm{V}_{G S}\right)$ between -2 and $+2 \mathrm{~V}$ with a step of $1 \mathrm{mV}$ and measures the associated currents $I_{D S}$ and $I_{G S}$. All measurement are conducted in room condition (temperature and pressure).

\section{Supporting Informations}

The Supporting Information is available free of charge on the ACS Publications website at DOI:

Size determination of nanoparticles, $X$-ray diffractogram, Tauc plot, process and effect of POM grafting, field effect transistor measurements and photoconduction under UV illumination

\section{ACKNOWLEDEGMENTS}

We thank C. Delerue for valuable discussion on the effect of the charge distribution. EL thanks the support ERC starting grant blackQD (grant $n^{\circ}$ 756225). We acknowledge the use of clean-room facilities from the "Centrale de Proximité Paris-Centre". This work has been supported by the Region lle-de-France in the framework of DIM Nano-K (grant dopQD). This work was supported by French state funds managed by the ANR within the Investissements d'Avenir programme under reference ANR-11-IDEX-0004-02, and more specifically within the framework of the Cluster of Excellence MATISSE and also by the grant Nanodose and IPER-Nano2. JQ thanks the Chinese Scholar council for PhD grant while NG and JR thank Nexdot for post doctorate funding.

\section{REFERENCES}

${ }^{1}$ Ackerman, M. M.; Tang, X.; Guyot-Sionnest, P. Fast and Sensitive Colloidal Quantum Dot Mid-Wave Infrared Photodetectors. ACS Nano 2018, 12, 7264-7271.

${ }^{2}$ Tang, X.; Ackerman, M. M.; Guyot-Sionnest, P. Thermal Imaging with Plasmon Resonance Enhanced HgTe Colloidal Quantum Dot Photovoltaic Devices. ACS Nano 2018, 12, 7362-7370.

${ }^{3}$ Cryer, M. E.; Halpert, J. E. $300 \mathrm{~nm}$ Spectral Resolution in the Mid-Infrared with Robust, High Responsivity Flexible Colloidal Quantum Dot Devices at Room Temperature. ACS Photonics 2018, 5, 3009-3015.

${ }^{4}$ Jagtap, A.; Martinez, B.; Goubet, N.; Chu, A.; Livache, C.; Gréboval, C.; Ramade, J.; Amelot, D.; Trousset, P.; Triboulin, A.; Ithurria, S.; Silly, M.G.; Dubertret, B.; Lhuillier, E. Design of a Unipolar Barrier for a Nanocrystal-Based Short-Wave Infrared Photodiode. ACS Photonics 2018, 5, 4569-4576.

${ }^{5}$ Li, L.; Reiss, P. One-Pot Synthesis of Highly Luminescent InP/ZnS Nanocrystals without Precursor Injection. J. Am. Chem. Soc. 2008, 130, 11588-11589.

${ }^{6}$ Mićić, O. I.; Sprague, J.; Lu, Z.; Nozik, A. J. Highly Efficient Band-edge Emission from InP Quantum Dots. Appl. Phys. Lett. 1996, 68, 3150-3152.

${ }^{7}$ Hines, M. A.; Scholes, G. D. Colloidal PbS Nanocrystals with Size-Tunable Near-Infrared Emission: Observation of PostSynthesis Self-Narrowing of the Particle Size Distribution. Adv. Mater. 2003, 15, 1844-1849.

${ }^{8}$ Bakueva, L.; Musikhin, S.; Hines, M. A.; Chang, T.-W. F.; Tzolov, M.; Scholes, G. D.; Sargent, E. H. Size-Tunable Infrared (1000-1600 nm) Electroluminescence from PbS Quantum-Dot Nanocrystals in a Semiconducting Polymer. Appl. Phys. Lett. 2003, 82, 2895-2897.

${ }^{9}$ Green, M.; Mirzai, H. Synthetic Routes to Mercury Chalcogenide Quantum Dots. J. Mater. Chem. C 2018, 6, 50975112. 
${ }^{10}$ Lhuillier, E.; Keuleyan, S.; Liu, H.; Guyot-Sionnest, P. Mid-IR Colloidal Nanocrystals. Chem. Mater. 2013, 25, $1272-$ 1282.

${ }^{11}$ Jagtap, A.; Livache, C.; Martinez, B.; Qu, J.; Chu, A.; Gréboval, C.; Goubet, N.; Lhuillier, E. Emergence of Intraband Transitions in Colloidal Nanocrystals [Invited]. Opt. Mater. Express 2018, 8, 1174-1183.

12 Jeong, K. S.; Deng, Z.; Keuleyan, S.; Liu, H.; Guyot-Sionnest, P. Air-Stable n-Doped Colloidal HgS Quantum Dots. J. Phys. Chem. Lett. 2014, 5, 1139-1143

${ }^{13}$ Deng, Z.; Jeong, K. S.; Guyot-Sionnest, P. Colloidal Quantum Dots Intraband Photodetectors. ACS Nano 2014, 8, 11707-11714.

${ }^{14}$ Kim, J.; Choi, D.; Jeong, K. S. Self-Doped Colloidal Semiconductor Nanocrystals with Intraband Transitions in Steady State. Chem. Commun. 2018, 54, 8435-8445.

${ }^{15}$ Goubet, N.; Jagtap, A.; Livache, C.; Martinez, B.; Portalès, H.; Xu, X. Z.; Lobo, R. P. S. M.; Dubertret, B.; Lhuillier, E. Terahertz HgTe Nanocrystals: Beyond Confinement. J. Am. Chem. Soc. 2018, 140, 5033-5036.

${ }^{16}$ Sahu, A.; Qi, L.; Kang, M. S.; Deng, D.; Norris, D. J. Facile Synthesis of Silver Chalcogenide $\left(\mathrm{Ag}_{2} \mathrm{E} ; \mathrm{E}=\mathrm{Se}, \mathrm{S}, \mathrm{Te}\right)$ Semiconductor Nanocrystals. J. Am. Chem. Soc. 2011, 133, 6509-6512.

${ }^{17}$ Sahu, A.; Khare, A.; Deng, D. D.; Norris, D. J. Quantum Confinement in Silver Selenide Semiconductor Nanocrystals. Chem. Commun. 2012, 48, 5458-5460.

${ }_{18}$ Park, M.; Choi, D.; Choi, Y.; Shin, H.; Jeong, K. S. Mid-Infrared Intraband Transition of Metal Excess Colloidal Ag2Se Nanocrystals. ACS Photonics 2018, 5, 1907-1911.

${ }^{19}$ Qu, J.; Goubet, N.; Livache, C.; Martinez, B.; Amelot, D.; Gréboval, C.; Chu, A.; Ramade, J.; Cruguel, H.; Ithurria, S.; Silly, M.G.; Lhuillier, E. Intraband Mid-Infrared Transitions in $\mathrm{Ag}_{2} \mathrm{Se}$ Nanocrystals: Potential and Limitations for Hg-Free Low-Cost Photodetection. J. Phys. Chem. C 2018, 122, 18161-18167.

${ }^{20}$ Agrawal, A.; Cho, S. H.; Zandi, O.; Ghosh, S.; Johns, R. W.; Milliron, D. J. Localized Surface Plasmon Resonance in Semiconductor Nanocrystals. Chem. Rev. 2018, 118, 3121-3207.

${ }^{21}$ Agrawal, A.; Johns, R. W.; Milliron, D. J. Control of Localized Surface Plasmon Resonances in Metal Oxide Nanocrystals. Annu. Rev. Mater. Res. 2017, 47, 1-31.

${ }^{22}$ Diroll, B. T.; Gordon, T. R.; Gaulding, E. A.; Klein, D. R.; Paik, T.; Yun, H. J.; Goodwin, E. D.; Damodhar, D.; Kagan, C. R.; Murray, C. B. Synthesis of N-Type Plasmonic Oxide Nanocrystals and the Optical and Electrical Characterization of Their Transparent Conducting Films. Chem. Mater. 2014, 26, 4579-4588.

${ }^{23}$ Della Gaspera, E.; Bersani, M.; Cittadini, M.; Guglielmi, M.; Pagani, D.; Noriega, R.; Mehra, S.; Salleo, A.; Martucci, A. Low-Temperature Processed Ga-Doped ZnO Coatings from Colloidal Inks. J. Am. Chem. Soc. 2013, 135, 3439-3448.

${ }^{24}$ Buonsanti, R.; Llordes, A.; Aloni, S.; Helms, B. A.; Milliron, D. J. Tunable Infrared Absorption and Visible Transparency of Colloidal Aluminum-Doped Zinc Oxide Nanocrystals. Nano Lett. 2011, 11, 4706-4710.

${ }^{25}$ Ghosh, S.; Saha, M.; De, S. K. Tunable Surface Plasmon Resonance and Enhanced Electrical Conductivity of In Doped ZnO Colloidal Nanocrystals. Nanoscale 2014, 6, 7039-7051.

${ }^{26}$ Ito, D.; Yokoyama, S.; Zaikova, T.; Masuko, K.; Hutchison, J. E. Synthesis of Ligand-Stabilized Metal Oxide Nanocrystals and Epitaxial Core/Shell Nanocrystals via a Lower-Temperature Esterification Process. ACS Nano 2014, 8, 64-75.

27 Shanker, G. S.; Tandon, B.; Shibata, T.; Chattopadhyay, S.; Nag, A. Doping Controls Plasmonics, Electrical Conductivity, and Carrier-Mediated Magnetic Coupling in Fe and $\mathrm{Sn}$ Codoped $\mathrm{In}_{2} \mathrm{O}_{3}$ nanocrystals: Local Structure Is the Key. Chem. Mater. 2015, 27, 892-900.

${ }^{28}$ Tandon, B.; Shanker, G. S.; Nag, A. Multifunctional Sn- and Fe-Codoped $\ln _{2} \mathrm{O}_{3}$ colloidal Nanocrystals: Plasmonics and Magnetism. J. Phys. Chem. Lett. 2014, 5, 2306-2311.

${ }^{29}$ Zandi, O.; Agrawal, A.; Shearer, A. B.; Reimnitz, L. C.; Dahlman, C. J.; Staller, C. M.; Milliron, D. J. Impacts of Surface Depletion on the Plasmonic Properties of Doped Semiconductor Nanocrystals. Nat. Mater. 2018, 17, 710.

30 Kanehara, M.; Koike, H.; Yoshinaga, T.; Teranishi, T. Indium Tin Oxide Nanoparticles with Compositionally Tunable Surface Plasmon Resonance Frequencies in the Near-IR Region. J. Am. Chem. Soc. 2009, 131, 17736-17737.

${ }^{31}$ Manthiram, K.; Alivisatos, A. P. Tunable Localized Surface Plasmon Resonances in Tungsten Oxide Nanocrystals. J. Am. Chem. Soc. 2012, 134, 3995-3998.

${ }^{32}$ Staller, C. M.; Robinson, Z. L.; Agrawal, A.; Gibbs, S. L.; Greenberg, B. L.; Lounis, S. D.; Kortshagen, U. R.; Milliron, D. J. Tuning Nanocrystal Surface Depletion by Controlling Dopant Distribution as a Route Toward Enhanced Film Conductivity. Nano Lett. 2018, 18, 2870-2878.

${ }^{33}$ Khoshkhoo, M. S.; Joseph, Y.; Maiti, S.; Schreiber, F.; Chassé, T.; Scheele, M. Tunable Charge Transport in Hybrid Superlattices of Indium Tin Oxide Nanocrystals and Metal Phthalocyanines-Toward Sensing Applications. Adv. Mater. Interfaces 2018, 5, 1701623.

${ }^{34}$ Johns, R. W.; Bechtel, H. A.; Runnerstrom, E. L.; Agrawal, A.; Lounis, S. D.; Milliron, D. J. Direct Observation of Narrow Mid-Infrared Plasmon Linewidths of Single Metal Oxide Nanocrystals. Nat. Commun. 2016, 7, 11583.

${ }^{35}$ Lounis, S. D.; Runnerstrom, E. L.; Bergerud, A.; Nordlund, D.; Milliron, D. J. Influence of Dopant Distribution on the Plasmonic Properties of Indium Tin Oxide Nanocrystals. J. Am. Chem. Soc. 2014, 136, 7110-7116. 
${ }^{36}$ Jansons, A. W.; Hutchison, J. E. Continuous Growth of Metal Oxide Nanocrystals: Enhanced Control of Nanocrystal Size and Radial Dopant Distribution. ACS Nano 2016, 10, 6942-6951.

${ }^{37}$ Crockett, B. M.; Jansons, A. W.; Koskela, K. M.; Johnson, D. W.; Hutchison, J. E. Radial Dopant Placement for Tuning Plasmonic Properties in Metal Oxide Nanocrystals. ACS Nano 2017, 11, 7719-7728.

${ }^{38}$ Delerue, C. Minimum Line Width of Surface Plasmon Resonance in Doped ZnO Nanocrystals. Nano Lett. 2017, 17, 7599-7605.

${ }^{39}$ Wang, Y.; Runnerstrom, E. L.; Milliron, D. J. Switchable Materials for Smart Windows. Annu. Rev. Chem. Biomol. Eng. 2016, 7, 283-304.

${ }^{40}$ Mendelsberg, R. J.; McBride, P. M.; Duong, J. T.; Bailey, M. J.; Llordes, A.; Milliron, D. J.; Helms, B. A. Dispersible Plasmonic Doped Metal Oxide Nanocrystal Sensors That Optically Track Redox Reactions in Aqueous Media with SingleElectron Sensitivity. Adv. Opt. Mater. 2015, 3, 1293-1300.

${ }^{41}$ Lou, Z.; Gu, Q.; Liao, Y.; Yu, S.; Xue, C. Promoting Pd-Catalyzed Suzuki Coupling Reactions through near-Infrared Plasmon Excitation of WO3-x Nanowires. Appl. Catal. B Environ. 2016, 184, 258-263.

${ }^{42}$ Lhuillier, E.; Keuleyan, S.; Guyot-Sionnest, P. Optical Properties of HgTe Colloidal Quantum Dots. Nanotechnology 2012, 23, 175705.

${ }^{43}$ Moreels, I.; Lambert, K.; Smeets, D.; De Muynck, D.; Nollet, T.; Martins, J. C.; Vanhaecke, F.; Vantomme, A.; Delerue, C.; Allan, G.; Hens, Z. Size-Dependent Optical Properties of Colloidal PbS Quantum Dots. ACS Nano 2009, 3, 3023-3030.

${ }^{44}$ Anderson, L. J. E.; Mayer, K. M.; Fraleigh, R. D.; Yang, Y.; Lee, S.; Hafner, J. H. Quantitative Measurements of Individual Gold Nanoparticle Scattering Cross Sections. J. Phys. Chem. C 2010, 114, 11127-11132.

${ }^{45}$ Wang, C.; Shim, M.; Guyot-Sionnest, P. Electrochromic Nanocrystal Quantum Dots. Science 2001, 291, 2390-2392.

${ }^{46}$ Runnerstrom, E. L.; Llordés, A.; Lounis, S. D.; Milliron, D. J. Nanostructured Electrochromic Smart Windows: Traditional Materials and NIR-Selective Plasmonic Nanocrystals. Chem. Commun. 2014, 50, 10555-10572.

${ }^{a}$ Factor, and Carrier Mobility in $\mathrm{Cr}-\mathrm{Sn}$ Doped $\operatorname{In}_{2} \mathrm{O}_{3}$ Nanocrystals. Chem. Mater. 2017, 29, 9360-9368.

${ }^{48}$ Askari, S.; Mariotti, D.; Stehr, J. E.; Benedikt, J.; Keraudy, J.; Helmersson, U. Low-Loss and Tunable Localized MidInfrared Plasmons in Nanocrystals of Highly Degenerate InN. Nano Lett. 2018, 18, 5681-5687.

${ }^{49}$ Edwards, P. P.; Porch, A.; Jones, M. O.; Morgan, D. V.; Perks, R. M. Basic Materials Physics of Transparent Conducting Oxides. Dalton Trans. 2004, 0, 2995-3002.

${ }^{50}$ Staller, C. M.; Agrawal, A.; Gibbs, S. L.; Cabezas, C. A. S.; Johns, R. W.; Milliron, D. J. Quantitative Analysis of Semiconductor Nanocrystal Ensemble Optical Extinction. arXiv:1812.10142 [cond-mat, physics:physics] 2018.

${ }^{51}$ Turchi, I. J.; Dewar, M. J. S. Chemistry of oxazoles. Chem. Rev. 1975, 75, 389-437.

${ }^{52}$ Lhuillier, E.; Ithurria, S.; Descamps-Mandine, A.; Douillard, T.; Castaing, R.; Xu, X. Z.; Taberna, P.-L.; Simon, P.; Aubin, H.; Dubertret, B. Investigating the N- and p-Type Electrolytic Charging of Colloidal Nanoplatelets. J. Phys. Chem. C 2015, 119, 21795-21799.

${ }^{53}$ Lee, J.; Lee, S.; Li, G.; Petruska, M. A.; Paine, D. C.; Sun, S. A Facile Solution-Phase Approach to Transparent and Conducting ITO Nanocrystal Assemblies. J. Am. Chem. Soc. 2012, 134, 13410-13414.

${ }^{54}$ Samadi Khoshkhoo, M.; Maiti, S.; Schreiber, F.; Chassé, T.; Scheele, M. Surface Functionalization with Copper Tetraaminophthalocyanine Enables Efficient Charge Transport in Indium Tin Oxide Nanocrystal Thin Films. ACS Appl. Mater. Interfaces 2017, 9, 14197-14206.

${ }^{55}$ Guyot-Sionnest, P. Electrical Transport in Colloidal Quantum Dot Films. J. Phys. Chem. Lett. 2012, 3, 1169-1175.

${ }^{56}$ Liu, H.; Pourret, A.; Guyot-Sionnest, P. Mott and Efros-Shklovskii Variable Range Hopping in CdSe Quantum Dots Films. ACS Nano 2010, 4, 5211-5216.

${ }^{57}$ Martinez, B.; Livache, C.; Mouafo, L. D. N.; Goubet, N.; Keuleyan, S.; Cruguel, H.; Ithurria, S.; Aubin, H.; Ouerghi, A.; Doudin, B.; Lacaze, E.; Dubertret, B.; Silly, M. G.; Lobo, R.; Dayen, J-F.; Lhuillier, E. HgSe Self-Doped Nanocrystals as a Platform to Investigate the Effects of Vanishing Confinement. ACS Appl. Mater. Interfaces 2018, 10, 11880-11887.

${ }^{58}$ Moreira, H.; Yu, Q.; Nadal, B.; Bresson, B.; Rosticher, M.; Lequeux, N.; Zimmers, A.; Aubin, H. Electron Cotunneling Transport in Gold Nanocrystal Arrays. Phys. Rev. Lett. 2011, 107, 176803.

${ }^{59}$ Swisher, S. L.; Volkman, S. K.; Subramanian, V. Tailoring Indium Oxide Nanocrystal Synthesis Conditions for Air-Stable High-Performance Solution-Processed Thin-Film Transistors. ACS Appl. Mater. Interfaces 2015, 7, 10069-10075.

${ }^{60}$ Konstantatos, G.; Sargent, E. H. PbS Colloidal Quantum Dot Photoconductive Photodetectors: Transport, Traps, and Gain. Appl. Phys. Lett. 2007, 91, 173505.

61 Jin, Y.; Wang, J.; Sun, B.; Blakesley, J. C.; Greenham, N. C. Solution-Processed Ultraviolet Photodetectors Based on Colloidal ZnO Nanoparticles. Nano Lett. 2008, 8, 1649-1653.

${ }^{62}$ Hasan Farooqi, M. M.; Srivastava, R. K. Structural, Optical and Photoconductivity Study of ZnO Nanoparticles Synthesized by Annealing of ZnS Nanoparticles. J. Alloys Compd. 2017, 691, 275-286.

${ }^{63}$ Choi, J.; Jo, J. W.; Arquer, F. P. G. de; Zhao, Y.-B.; Sun, B.; Kim, J.; Choi, M.-J.; Baek, S.-W.; Proppe, A. H.; Seifitokaldani, A. Nam, D.-H.; Li, P.; Ouellette, O.; Kim, Y.; Voznyy, O.; Hoogland, S.; Kelley, S. O.; Lu, Z.; Sargent, E.H. Activated Electron-Transport Layers for Infrared Quantum Dot Optoelectronics. Adv. Mater. 2018, 30, 1801720. 
${ }^{64}$ Shen, G.; Guyot-Sionnest, P. HgS and HgS/CdS Colloidal Quantum Dots with Infrared Intraband Transitions and Emergence of a Surface Plasmon. J. Phys. Chem. C 2016, 120, 11744-11753.

${ }^{65}$ Schimpf, A. M.; Thakkar, N.; Gunthardt, C. E.; Masiello, D. J.; Gamelin, D. R. Charge-Tunable Quantum Plasmons in Colloidal Semiconductor Nanocrystals. ACS Nano 2014, 8, 1065-1072.

${ }^{66}$ Martinez, B.; Livache, C.; Meriggio, E.; Xu, X. Z.; Cruguel, H.; Lacaze, E.; Proust, A.; Ithurria, S.; Silly, M. G.; Cabailh, G.; Volatron, F.; Lhuillier, E. Polyoxometalate as Control Agent for the Doping in HgSe Self-Doped Nanocrystals. J. Phys. Chem. C 2018, 122, 26680-26685.

67 Martinez, B.; Livache, C.; Goubet, N.; Jagtap, A.; Cruguel, H.; Ouerghi, A.; Lacaze, E.; Silly, M. G.; Lhuillier, E. Probing Charge Carrier Dynamics to Unveil the Role of Surface Ligands in HgTe Narrow Band Gap Nanocrystals. J. Phys. Chem. C 2018, 122, 859-865.

${ }^{68}$ Livache, C.; Goubet, N.; Martinez, B.; Jagtap, A.; Qu, J.; Ithurria, S.; Silly, M. G.; Dubertret, B.; Lhuillier, E. Band Edge Dynamics and Multiexciton Generation in Narrow Band Gap HgTe Nanocrystals. ACS Appl. Mater. Interfaces 2018, 10, 11880-11887.

69 Lhuillier, E.; Robin, A.;Ithurria, S.; Aubin, H.; Dubertret B. Electrolyte-gated colloidal nanoplatelets-based phototransistor and its use for bicolor detection. Nano letters 2014, 14, 2715-271.

${ }^{70}$ Lhuillier, E.; Dayen, J.-F.; Thomas, D. O.; Robin, A.; Doudin, B.; Dubertret, B. Nanoplatelets Bridging a Nanotrench: A New Architecture for Photodetectors with Increased Sensitivity. Nano Lett. 2015, 15, 1736-1742.

${ }^{71}$ Souchay, P. Polyanions et polycations; Monographies de chimie minérale; Gauthier-Villars: Paris, 1963. 


\section{TOC graphic}

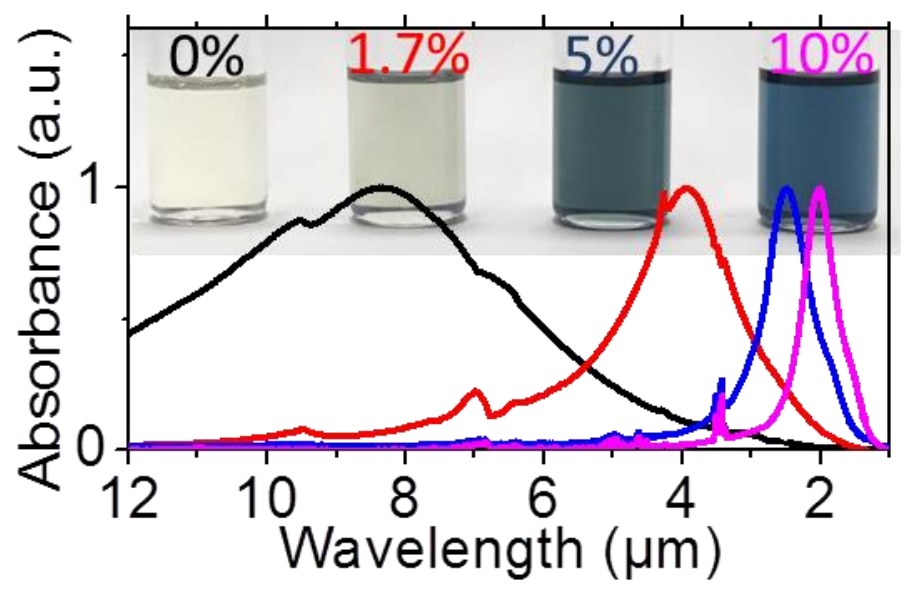

